\title{
Minimum Length Scheduling for Power Constrained Harvest-then-Transmit Communication Networks
}

\author{
Elif Dilek Salik, Aysun Gurur Onalan, and Sinem Coleri Ergen \\ Department of Electrical and Electronics Engineering \\ Koc University, Istanbul, Turkey \\ Email: $\{$ esalik, aonalan17, sergen $\} @$ ku.edu.tr
}

\begin{abstract}
We consider a wireless powered, harvest-thentransmit communication network, which consists of a single antenna, energy and information access point (AP) and multiple, single antenna, batteryless users with energy harvesting capabilities. At the beginning of a time frame, the AP broadcasts energy in the downlink to the users. Then, users transmit their data to the AP in the uplink, using their harvested energy. We formulate the optimization problem with the objective of minimizing the total schedule length, subject to the constraints on the minimum amount of data to be sent to the AP, and unlike previous studies, the maximum transmit power for the information transmission. This problem is nonlinear and non-convex. The solution is based on bi-level optimization, consisting of optimizing the transmit power allocation of the nodes for a given energy harvesting time and searching over harvesting time allocation. We also propose a heuristic algorithm in which we incorporate the optimal solution of a single user network. Simulation results demonstrate that under appropriate network conditions, our proposed algorithms provide close-to-optimal results with a reasonable run time compared to another time minimization algorithm which does not integrate the uplink power constraint.
\end{abstract}

\section{INTRODUCTION}

$5 \mathrm{G}$ enables massive machine-to-machine (M2M) communication with a total of 50 billion connected devices by 2020 [1]. A drawback for M2M communication is that devices are usually powered by batteries with limited capacity. Renewing batteries may not be feasible due to the operating conditions of a network (e.g., body implants, toxic environment, large scale networks) A promising, environment independent and fully controllable solution is radio frequency (RF) energy harvesting $(\mathrm{EH})$, in which energy is harvested from the propagating electromagnetic waves. Hence, RF-EH is suitable for M2M applications with critical QoS and delay constraints, such as healthcare, automotive, security etc.

The joint design of downlink (DL) energy transfer and uplink (UL) information transmission (IT) in wireless powered communication networks (WPCNs) has been investigated for throughput maximization [2]-[5]. Harvest-then-transmit (HTT) network with multiple users under half [2] and full [3] duplex AP, the utilization of information and energy beamformings for multiple antenna networks [4], [5] are studied. [6] imposes a maximum UL power limit on users while maximizing energy

This work is supported by Scientific and Technological Research Council of Turkey Grant \#117E241. efficiency. However, none of these works utilized the EH and IT lengths to meet the delay requirements.

While increasing EH and IT lengths has a positive effect in the throughput performance, there exists a trade-off between the harvested energy, throughput, and delay [2]. In the context of tradeoff, when to switch between EH and IT [7], the coupling effect between EH of a user and ITs of the previously assigned users [8], the coupling between consecutively scheduled users in a chain model [9], and a half-duplex HTT network [10] are studied for total time minimization. Although the transmit power of a device is restricted by its hardware in practice, aforementioned works allowed the users to transmit at any power. To the best of our knowledge, there is no work in the literature recognizing the UL transmit power limit while minimizing the total transmission time.

In this paper, we consider a HTT network with multiple EH users and a half-duplex AP, deploying TDMA. We jointly optimize DL and UL time and UL power allocations to minimize the total scheduling length of the network. The problem is constrained by the data amounts that each user has to send in the $\mathrm{UL}$ and the maximum UL transmit power. This is a nonlinear and non-convex problem and we propose a logarithmic time algorithm that provides the optimal solution within an error bound following the derivations of the optimality conditions. And, we propose a suboptimal algorithm, employing the optimal solution we derive for a single user network.

The paper is organized as follows: Section II describes the system model and the assumptions used throughout the paper. Section III formulates the minimum length scheduling problem. Section IV provides the near optimal solution with the associated algorithm. The suboptimal algorithm is given in Section V. Section VI presents simulations and performance evaluation. Concluding remarks are given in Section VII.

\section{System Model AND Assumptions}

- We considered a HTT network with $K$ users, $U_{i}, i=$ $1, \ldots, K$, and one AP, all are equipped with single antenna and operating over the same frequency.

- In a transmission frame, AP broadcasts a DL signal to the users for the first $\tau_{0}$ amount of time for $\mathrm{EH}$, while $\tau_{i}$ amount of time is assigned for the IT from $U_{i}$ to the AP, later. The users equip neither any other energy source nor 
battery to store the harvested energy for latter use; thus, all the EH and ITs take place within a frame.

- The AP maintains the synchronization of the network and informs the users about the current schedule at the beginning of each time frame. This signaling is assumed to be fixed length and negligible.

- The channel power gains from the AP to $U_{i}$ in the DL and from $U_{i}$ to the AP in the UL are denoted by $h_{i}$ and $g_{i}$, respectively. The channels are block-fading and gains are perfectly known at the AP.

- $U_{i}$ has to send a certain amount of data in a frame, denoted as $D_{i}$, which is constant and non-negative.

- AP transmits with constant power $P_{A}$ in the DL, which is sufficiently large such that the energy harvested due to the receiver noise is negligible. Then, the total energy harvested by $U_{i}$ is $E_{i}=\zeta_{i} P_{A} h_{i} \tau_{0}$, where $\zeta_{i} \in(0,1)$ is the EH efficiency of $U_{i}$, and assumed to be constant in this paper.

- Each $U_{i}$ uses a portion of its harvested energy, $\eta_{i}$, in the UL, $0<\eta_{i} \leq 1$. Continuous power model is assumed and the average transmit power of $U_{i}$ in the UL for IT, $P_{i}$, is given by

$$
P_{i}=\eta_{i} \frac{E_{i}}{\tau_{i}}=\frac{\eta_{i} \zeta_{i} P_{A} h_{i} \tau_{0}}{\tau_{i}} .
$$

- There is an upper limit on the power radiated by $U_{i}$, mostly due to the hardware limitations of the power amplifier, denoted as $P_{i}^{\max }$.

- There is no interference during IT. The instantaneous UL transmission rate for $U_{i}$ is

$$
R_{i}\left(\tau, \eta_{i}\right)=W \log _{2}\left(1+\frac{g_{i} \eta_{i} \zeta_{i} P_{A} h_{i} \tau_{0}}{\tau_{i} \sigma^{2}}\right),
$$

where $\tau=\left[\tau_{0} \tau_{1} \ldots \tau_{K}\right], W$ is the channel bandwidth and $\sigma^{2}$ is the power of Additive White Gaussian Noise (AWGN) at the AP.

\section{Optimization Problem}

The optimization of transmit power and time allocation to minimize the scheduling length of a HTT WPCN with one AP and $\mathrm{K}$ users, subject to the constraints that each user has a certain amount of data to send to the AP with a maximum allowable transmit power is mathematically formulated.

The goal of the optimization problem is to minimize the sum of the EH and IT lengths. (3b) represents the data requirement that $U_{i}$ has to meet in each frame. (3c) states that the transmit power of $U_{i}$ cannot exceed a maximum allowable power. (3d) defines the range of $\eta_{i}$, serving to adjust $U_{i}$ 's UL transmit power. $(3 \mathrm{e})$ is the non-negativity constraint for the time allocation variables.

$$
\begin{array}{ll}
\min _{\tau, \eta} & \sum_{i=0}^{K} \tau_{i} \\
\text { s.t. } & \tau_{i} W \log _{2}\left(1+\frac{g_{i} \eta_{i} \zeta_{i} P_{A} h_{i} \tau_{0}}{\tau_{i} \sigma^{2}}\right) \geq D_{i}, \forall i=1, \ldots, K, \\
& \frac{\eta_{i} \zeta_{i} P_{A} h_{i} \tau_{0}}{\tau_{i}} \leq P_{i}^{\max }, \quad \forall i=1, \ldots, K \\
& 0<\eta_{i} \leq 1, \quad \forall i=1, \ldots, K \\
& \tau_{i} \geq 0, \quad \forall i=0, \ldots, K .
\end{array}
$$

Lemma 1. $P_{i}$, defined in (1) is a nonlinear and neither convex nor concave function of $\left(\tau, \eta_{i}\right)$.

Proof. Let $\nabla^{2} P_{i}\left(\tau, \eta_{i}\right)$ denote the Hessian of $P_{i}\left(\tau, \eta_{i}\right)$ defined in (1). Then, the eigenvalues of $\nabla^{2} P_{i}\left(\tau, \eta_{i}\right)$, denoted as $\lambda_{\nabla^{2} P_{i}}$, are

$$
\lambda_{\nabla^{2} P_{i}}=\left[\begin{array}{c}
0 \\
\frac{A_{i}\left(\left(\tau_{0}^{2}+\tau_{i}^{2}\right)\left(\eta_{i}^{2}+\tau_{i}^{2}\right)\right)^{\frac{1}{2}}+A_{i} \eta_{i} \tau_{0}}{\tau_{i}^{3}} \\
\frac{-A_{i}\left(\left(\tau_{0}^{2}+\tau_{i}^{2}\right)\left(\eta_{i}^{2}+\tau_{i}^{2}\right)\right)^{\frac{1}{2}}+A_{i} \eta_{i} \tau_{0}}{\tau_{i}^{3}}
\end{array}\right]
$$

where $A_{i}=\zeta_{i} P_{A} h_{i}$. Since $A_{i}, \tau_{i}$ and $\eta_{i}>0$ for all $i=$ $1, \ldots, K$, and $\tau_{0}>0, \lambda_{\nabla^{2} P_{i}}(2,1)>0$ and $\lambda_{\nabla^{2} P_{i}}(3,1)<0$. The last inequality follows from $A_{i}\left(\left(\tau_{0}^{2}+\tau_{i}^{2}\right)\left(\eta_{i}^{2}+\tau_{i}^{2}\right)\right)^{\frac{1}{2}}>A_{i} \eta_{i} \tau_{0}$. Therefore, $\nabla^{2} P_{i}\left(\tau, \eta_{i}\right)$ is neither a semidefinite positive nor a semidefinite negative matrix. Consequently, $P_{i}\left(\tau, \eta_{i}\right)$ is neither a convex nor a concave function of $\left(\tau, \eta_{i}\right)$.

From Lemma 1, it follows that the equation (3c) does not represent a convex set. Hence, the optimization problem is nonlinear and non-convex.

\section{Optimal Algorithm}

Problem (3) is difficult to solve because of its non-convexity and nonlinearity. To achieve the optimal solution, we transform the problem into bi-level optimization. We treat the $K$-user network as $K$ networks with a single user and find an expression for the individual IT times of the users for a fixed EH time. Then, the optimal EH time can be searched with bisection method as the sum of the EH and IT lengths are proven to be convex over EH time.

\section{A. Optimal Solution}

Problem (3) is separable into $K$ easy and convex subproblems with the objective function and the variable $\tau_{i}$ and constraints regarding only $U_{i}$ for all $i=1, \ldots, K$, where $\tau_{0}$ is the only coupling variable between subproblems. Then, the optimal solution to the master problem on $\tau_{0}$ with the objective function as the sum of the objectives of the subproblems and $\tau_{0}$ gives the optimal solution to the original problem [11].

All of the subproblems are in the same reduced form of Problem (3) for $K=1$ and fixed $\tau_{0}$. To solve these subproblems, we exploit the nature of a single user network. Note that, for $K=1, \tau_{0}$ and $\tau_{1}$ is adjusted just to meet $U_{1}$ 's requirements. Thus, only the amount of energy required for the UL IT is harvested, i.e., $\eta_{1}=1$.

- For $K=1$ and $\eta_{1}=1$ (or fixed $\left.\tau_{0}\right), R\left(\left[\tau_{0}, \tau_{1}, \eta_{1}\right]\right.$ ) defined in (2), turns into a nonlinear, concave function of $\left[\tau_{0}, \tau_{1}\right]$ (or of $\left[\tau_{1}, \eta_{1}\right]$ ) [2], (3b) represents a convex set, and the objective function and (3c) are linear. Thus, both of these reduced problems of (3) are nonlinear and convex.

- Due to the convexity of the reduced problems and their linear objective functions, their optimal solutions will lie on the boundary of their feasible region [12] and satisfy (3b) with equality [8]. 
Definition 1. $\tau_{0}^{l}$ and $\tau_{1}^{l}$ denote the values of $\tau_{0}$ and $\tau_{1}$, respectively, as the solution to the system consisting of (3b) and $(3 c)$, both at equality, for $K=1$ and $\eta_{1}=1$,

$$
\begin{gathered}
\tau_{0}^{l}=\frac{P_{1}^{\max } D_{1}}{W \zeta_{1} P_{A} h_{1} \log _{2}\left(\frac{P_{1}^{\text {max }} \gamma_{1}}{\zeta_{1} P_{A} h_{1}}+1\right)}, \\
\tau_{1}^{l}=\frac{D_{1}}{W \log _{2}\left(\frac{P_{1}^{\text {max }} \gamma_{1}}{P_{A} \zeta_{1} h_{1}}+1\right)} .
\end{gathered}
$$

In the system assumed in Definition $1, U_{1}$ transmits at $P_{1}^{\max }$ in the UL. Hence, $\tau_{0}^{l}$ represents a critical point for the case where $\tau_{0}$ is fixed, and $\eta_{1}$ is varying. Any $\tau_{0}>\tau_{0}^{l}$ will result in an excess amount of energy for $U_{1}$, and $\eta_{1}$ is adjusted to optimize UL transmit power while keeping $\tau_{1}$ the same, as opposed to the lower $\tau_{0}$, where $U_{1}$ uses all the harvested energy and $\tau_{1}$ is increased to meet the data constraint. This is more formally expressed as follows:

Lemma 2. Problem (3) is solved for $\tau_{0}=\overline{\tau_{0}}$ and $K=1$. For $\overline{\tau_{0}} \geq \beta_{1}$, where $\beta_{1}=\tau_{0}^{l}$, the optimal solution is

$$
\begin{gathered}
\overline{\eta_{1}}=\frac{P_{1}^{\max }}{\zeta_{1} P_{A} h_{1} \overline{\tau_{0}}} \overline{\tau_{1}}, \\
\overline{\tau_{1}}=\tau_{1}^{l} .
\end{gathered}
$$

For $\overline{\tau_{0}}<\beta_{1}, \overline{\eta_{1}}$ is fixed to 1 , the optimization problem is reduced to a nonlinear equation,

$$
\frac{\left(2^{D_{1} / W \overline{\tau_{1}}}-1\right) \overline{\tau_{1}}}{\gamma_{1}}=\overline{\tau_{0}}
$$

where $\gamma_{1}=\frac{g_{1} \zeta_{1} P_{A} h_{1}}{\sigma^{2}}$.

Proof. The problem has two decision variables, but, $\eta_{1}$ is redundant and the trade off between $\tau_{1}$ and $\eta_{1}$ is irrelevant.

The solution to the system consisting of (3b) and (3c) for fixed $\tau_{0}$ is (7) and (8). By substituting (8) into (7) and solving it for $\overline{\tau_{0}}$, we get $\overline{\tau_{0}}=\beta_{1}$ as expected. If $\overline{\tau_{0}}<\beta_{1}$ in (7), then $\eta_{1}>1$, and this corner point is not feasible any more; otherwise, the optimal solution is found.

If the solution above is infeasible, then $\eta_{1}$ is fixed to 1 , forcing all harvested energy to be used in the IT. Hence, the optimal solution is found on the intersection point of (3b) with $\eta_{1}=1$. We rewrite (3b) such that $\eta_{1}$ is a function of $\tau_{1}$ and equate it to 1 , and get the expression in (9). Since, the solution to (9) is feasible already, it becomes the optimal solution to the problem.

Theorem 1. Let $g\left(\overline{\tau_{0}}\right)=\overline{\tau_{0}}+\sum_{i} \overline{\tau_{i}}\left(\overline{\tau_{0}}\right) \cdot g\left(\overline{\tau_{0}}\right)$ is convex over $\overline{\tau_{0}}$.

Proof. We prove that $\overline{\tau_{1}}\left(\overline{\tau_{0}}\right)$ is convex. Then, $g\left(\overline{\tau_{0}}\right)$ is also convex as $\overline{\tau_{i}}\left(\overline{\tau_{0}}\right) \mathrm{s}$ are all in the same form, and the convexity is closed under sum.

By Theorem 2.4.1(iii) in [13], $\overline{\tau_{1}}\left(\overline{\tau_{0}}\right)$ is convex if (1) $\overline{\tau_{1}}\left(\overline{\tau_{0}}\right)$ is proper, (2) the domain of the function denoted by $\operatorname{dom} \overline{\tau_{1}}$, i.e. the set of all possible $\overline{\tau_{0}}$, is convex, and (3) $\overline{\tau_{1}}\left(\overline{\tau_{0}}\right)$ is subdifferentiable at every $\overline{\tau_{0}} \in d o m \overline{\tau_{1}}$.
1) $\overline{\tau_{1}}\left(\overline{\tau_{0}}\right):(0,+\infty) \rightarrow(0,+\infty)$, is proper, since $\operatorname{dom} \overline{\tau_{1}}=$ $(0,+\infty) \neq \emptyset$.

2) $\operatorname{dom} \overline{\tau_{1}}$ is convex.

3) $\partial \overline{\tau_{1}}\left(\overline{\tau_{0}}\right)$ represents the subdifferential of $\overline{\tau_{1}}$ at $\overline{\tau_{0}}$, and it is defined as $\partial \overline{\tau_{1}}\left(\overline{\tau_{0}}\right)=\left\{m \mid m^{T}\left(y-\overline{\tau_{0}}\right) \leq \overline{\tau_{1}}(y)-\right.$ $\left.\overline{\tau_{1}}\left(\overline{\tau_{0}}\right), \forall y \in d o m \overline{\tau_{1}}\right\}$ and $\operatorname{dom} \partial \overline{\tau_{1}}(x)=\{x \in$ $\left.\operatorname{dom} \overline{\tau_{1}} \mid \partial \overline{\tau_{1}} \neq \emptyset\right\}$.

For $\overline{\tau_{0}} \neq \beta_{1}, \overline{\tau_{1}}$ is differentiable. Hence, the subdifferential of the function exists, it is unique and equal to the derivative of the function.

At $\overline{\tau_{0}}=\beta_{1}$, the derivative does not exist. To determine $\partial \overline{\tau_{1}}\left(\beta_{1}\right)$, we calculate subgradients of $\overline{\tau_{1}}$ at $\beta_{1}$, since $\partial \bar{\tau}_{1}\left(\beta_{1}\right)$ is the set of all subgradients, $m$ 's, defined by the inequality $m\left(y-\beta_{1}\right) \leq \overline{\tau_{1}}(y)-\overline{\tau_{1}}\left(\beta_{1}\right)$ for all $y$.

For $y \geq \beta_{1}, \overline{\tau_{1}}(y)$ is a constant function and the subgradient inequality is equivalent to $m\left(y-\beta_{1}\right) \leq 0$. $\left(y-\beta_{1}\right)>0$, then, $m \leq 0$.

For $y<\beta_{1}, \overline{\tau_{1}}(y)$ is a decreasing function, thus, $\overline{\tau_{1}}(y)-$ $\overline{\tau_{1}}\left(\beta_{1}\right)>0$. Let $\mu_{1}<0$ be a variable that is equal to $\frac{\overline{\tau_{1}}(y)-\overline{\tau_{1}}\left(\beta_{1}\right)}{y-\beta_{1}}$. Then, $m \geq \mu_{1}$.

Therefore, we have $\partial \overline{\tau_{1}}\left(\beta_{1}\right)=\left[\mu_{1}, 0\right]$. That means $\overline{\tau_{1}}$ is subdifferentiable at every $x \in \operatorname{dom} \overline{\tau_{1}}$, i.e., $\operatorname{dom} \overline{\tau_{1}}=$ $\operatorname{dom} \partial \overline{\tau_{1}}$.

In consequence of (1)-(3), $\overline{\tau_{1}}\left(\overline{\tau_{0}}\right)$ is convex. Thus, $g\left(\overline{\tau_{0}}\right)$, the sum of convex functions, is convex.

\section{B. Algorithm}

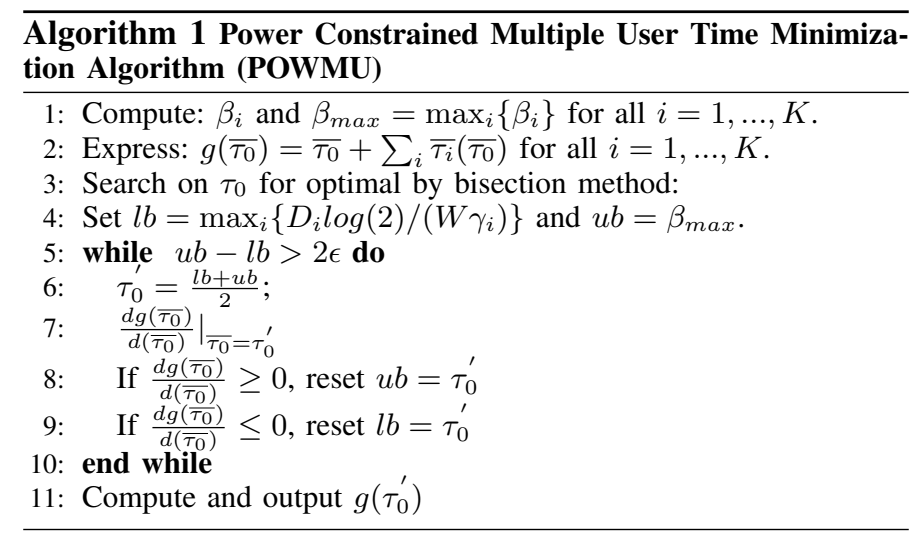

POWMU, first, calculates the critical points of $\overline{\tau_{i}}\left(\overline{\tau_{0}}\right), \beta_{i}$, for each $i=1, \ldots, K$. Next, the maximum of the critical points, $\beta_{\text {max }}$, is found. Total transmission time, $g\left(\overline{\tau_{0}}\right)$, is expressed. We perform a bisection search on $g\left(\overline{\tau_{0}}\right)$. The idea is to iteratively converge to the optimal solution inside a search interval by evaluating the slope of $g\left(\overline{\tau_{0}}\right)$ to check where the solution lies. When $\overline{\tau_{0}}>\beta_{i}, \overline{\tau_{i}}\left(\overline{\tau_{0}}\right)$ is constant for all $i$. Since the total transmit time is the sum of $\tau_{0}$ with $\overline{\tau_{i}}\left(\overline{\tau_{0}}\right)$ for all $i, \beta_{\max }$ is set as the upper bound, $u b$, of the search interval. The lower bound, $l b$, is set to $\max _{i}\left\{D_{i} \log (2) /\left(W \gamma_{i}\right)\right\}$ [10]. The current solution, $\tau_{0}^{\prime}$, is set to the geometric mean of the $l b$ and $u b$. If the first derivative of $g\left(\overline{\tau_{0}}\right)$ calculated at $\tau_{0}^{\prime}$ is nonnegative, meaning that the function increases at that point, then the $u b$ is reset to $\tau_{0}^{\prime}$. Otherwise, the $l b$ is reset to $\tau_{0}^{\prime}$. This procedure 
continues until the difference between $u b$ and $l b$ is less than $2 \epsilon$. Then, search stops and the scheduling length $g\left(\overline{\tau_{0}}\right)$ computed at the last $\tau_{0}^{\prime}$ is returned as the optimal solution.

Theorem 2. Given the initial $l b$ and $u b$, by bisection search method, to obtain an optimal solution in the error bound, $\epsilon$, the number of iterations, $k$, should be at least as large as $\log _{2}(u b-$ lb) $-\log _{2} \epsilon$.

Proof. Please refer to [14].

With the derivative calculations, $\mathcal{O}(K)$, at each iteration and by Theorem 2, the worst case performance of bisection search is $\mathcal{O}(K \log n)$, where $n$ is the length of the search array. In general, $n>>K$ and search also dominates $\mathcal{O}(K)$ calculations in the rest of the algorithm. Therefore, the overall complexity of POWMU is logarithmic.

\section{Heuristic Algorithm}

We propose a heuristic algorithm, MAX-EH, which provides a suboptimal solution to the multiple user problem by exploiting the optimality conditions of a single user network. MAX-EH finds $K$-many optimal EH times for $K$ users as if there are $K$ networks with a single user. The maximum among them is set as the network's EH time and having EH length known, IT lengths of each user are calculated accordingly.

\section{A. Analytical Analysis}

We have already started solving the Problem (3) for $K=1$ and $\eta_{1}=1$ in Section IV-A and stated in Lemma 2 that, for $\eta_{1}=1$, the problem is reduced to (9). Next, we will solve (9).

Theorem 3. The solution to the optimization problem with the objective $\left(\tau_{0}+\tau_{1}\right)$ and the constraints $(3 b),(3 e)$, and $\eta_{1}=1$, denoted as $\tau_{0}^{k}$ and $\tau_{1}^{k}$, given by

$$
\begin{gathered}
\tau_{1}^{k}=\frac{D_{1} \log (2)}{W \alpha_{1}}, \\
\tau_{0}^{k}=\frac{D_{1} \log 2}{W \alpha_{1} \gamma_{1}}\left(10^{\alpha_{1}}-1\right) .
\end{gathered}
$$

where $\alpha_{1}=\mathcal{W}\left(\frac{\gamma_{1}-1}{e}\right)+1$ and $\mathcal{W}(\cdot)$ is the Lambert W-Function.

Proof. Sketch of the proof is as follows. We will first rewrite (3b) such that $\tau_{0}$ is a function of $\tau_{1}$, which is, in fact, the same with (9) and it will be denoted as $V\left(\tau_{1}\right)$. Next, we will prove that $V\left(\tau_{1}\right)$ is strictly decreasing with respect to $\tau_{1}$. When we think of the objective function in the slope-intercept form, e.g. $\tau_{0}=-\tau_{1}+C_{1}$ and $C_{1}$ is the total transmission time that we want to minimize, one of the lines from the family of parallel lines constructed in this way forms a tangent line to $V\left(\tau_{1}\right)$. The tangent line is unique since $V\left(\tau_{1}\right)$ is strictly decreasing; and the tangent point minimizes the objective function. Hence, $\tau_{1}$ and $\tau_{0}$ values corresponding to this unique tangent point are calculated.

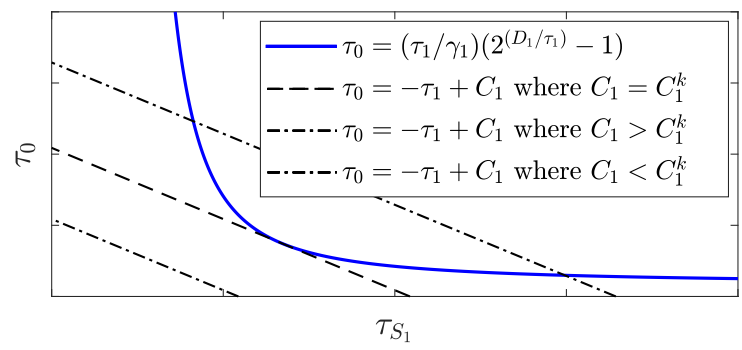

Fig. 1. Graphical representation of the constraint (3b).

First, we note that $\tau_{1}$ is strictly positive as to serve the strictly positive $D_{1}$. The first order derivative of $V\left(\tau_{1}\right)$ is

$$
\begin{array}{r}
\frac{d V\left(\tau_{1}\right)}{d \tau_{1}}=\frac{\left(2^{\frac{D_{1}}{W \tau_{1}}}\left(W \tau_{1}-D_{1} \log 2\right)-W \tau_{1}\right)}{W \gamma_{1} \tau_{1}} \\
<\frac{W \tau_{1}-D_{1} \log 2-W \tau_{1}}{W \gamma_{1} \tau_{1}}<0 .
\end{array}
$$

(12) indicates that the function $V\left(\tau_{1}\right)$ is strictly decreasing with respect to $\tau_{1}$.

We illustrate the graphical relationship between the curve $V\left(\tau_{1}\right)=\frac{\tau_{1}}{\gamma_{1}}\left(2^{\frac{D_{1}}{W \tau_{1}}}-1\right)$ and the line $\tau_{0}=-\tau_{1}+C_{1}$ in Fig. 1. Depending on the value of $C_{1}$, the line intersects with the curve either at two points, or at one unique point, or does not intersect at all.

Denote $C_{1}^{k}$ as the value of $C_{1}$ such that the line $\tau_{0}=-\tau_{1}+$ $C_{1}$ has only one intersection point with the curve $V\left(\tau_{1}\right)$, and the corresponding $\tau_{1}$, is denoted as $\tau_{1}^{k}$. Then, at the unique intersection point we have

$$
\begin{gathered}
\left.\frac{d V\left(\tau_{1}\right)}{d \tau_{1}}\right|_{\tau_{1}=\tau_{1}^{k}}=-1, \\
V\left(\tau_{1}^{k}\right)=-\tau_{1}^{k}+C_{1}^{k},
\end{gathered}
$$

From (13), $\tau_{1}^{k}$ is obtained as in (10). By (14), $C_{1}^{k}$ is obtained as

$$
C_{1}^{k}=\frac{D_{1} \log 2}{W \alpha_{1}}\left(1+\frac{10^{\alpha_{1}}-1}{\gamma_{1}}\right) .
$$

The area above the curve, $\tau_{1} \geq \frac{\tau_{1}}{\gamma_{1}}\left(2^{\frac{D_{1}}{W \tau_{1}}}-1\right)$, represents the feasible region on Fig. 1, accordingly,

- When $C_{1}<C_{1}^{k}$, there is no intersection between the line and the curve, hence, feasibility is not satisfied.

- When $C_{1}>C_{1}^{k}$, there are two intersection points between the line and the curve.

These derivations and observations prove that the objective is minimized at the unique intersection point of the line and the curve, when $C_{1}=C_{1}^{k}$.

Corollary 1. The optimal solution to the Problem (3) for $K=1$ is $\left(\tau_{0}^{k}, \tau_{1}^{k}\right)$ if this corner point solution satisfies the constraint (3c), i.e. it is feasible, by Theorem 3. Otherwise, the optimal solution is $\left(\tau_{0}^{l}, \tau_{1}^{l}\right)$ given in Definition 1, which is always feasible. 


\section{B. MAX-EH Algorithm}

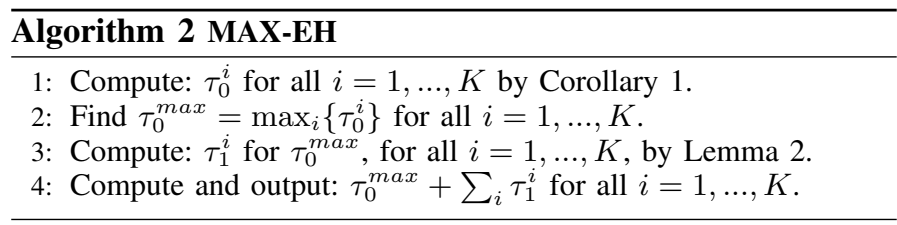

MAX-EH Algorithm is described in detail as follows: Individual optimal EH times of the users are calculated using Theorem 1 , and stored in $\tau_{0}^{i}$ for all $i=1, \ldots, K$. Then, their maximum is found and stored in $\tau_{0}^{\max }$. This maximum value is set as the network's EH length. Using $\tau_{0}^{\max }$, individual IT lengths of the users are calculated. Their sum with $\tau_{0}^{\max }$ is computed as total scheduling length of the network and set as the output. MAX-EH is a linear time algorithm with $\mathcal{O}(K)$.

\section{Simulations AND Numerical RESUlts}

The goal of the simulations is to compare the performances of the proposed algorithms, POWMU and MAX-EH, to Transmission Completion Time-minimized (TCT) Algorithm [10] and to evaluate the effect of the maximum power constraint on the network's performance.

TCT finds the optimal schedule length for HTT network where the users are free to transmit at any power. Hence, TCT might set transmit power of the users higher than $P_{\max }$ to shorthen IT lengths. In our simulations, we force TCT to obey $P_{\max }$ limitation, i.e., for any user allocated to transmit at a power level greater than $P^{\max }$, its transmit power is adjusted to $P^{\max }$

Simulation results are obtained by averaging the performance over 1000 independent random network topologies in MATLAB. The users are uniformly distributed within a circular area of radius $1 \mathrm{~m}$ and the AP is located at the center. The network is simulated for an indoor environment operating at a center frequency of $f_{c}=915 \mathrm{MHz}$ and a bandwidth of $W=1 \mathrm{MHz}$.

The channel attenuation is determined considering both large and small scale statistics. The attenuation due to the large scale statistics is modeled as $P L(d)=P L\left(d_{0}\right)+10 \alpha \log \left(d / d_{0}\right)+Z$, where $d$ is the distance between the user and the AP, $P L(d)$ is the path loss at distance $d$ in decibels, $P L\left(d_{0}\right)$ is the path loss at reference distance $d_{0}=1 \mathrm{~m}, \alpha$ is the path loss exponent [15] and $\mathrm{Z}$ is a Gaussian random variable with zero mean and standard deviation $\sigma_{z}$. The small-scale fading is modeled by Rayleigh fading with scale parameter $\Omega$ set to the mean power level determined by $P L(d)$ [16].

The simulation parameters are as follows: $\sigma^{2}=-50 \mathrm{dBm}$, $\sigma_{Z}=2 \mathrm{~dB}, P L\left(d_{0}\right)=31.7 \mathrm{~dB}, \alpha=2, \zeta=0.5, D=50$ bits, $P_{A P}=4 \mathrm{~W}$, and $P^{\max }=1 \mathrm{~mW}$.

Fig. 2 depicts the schedule length of a 5-user network for varying $P_{A}$. POWMU reduces the total time up to $4.1 \%$, compared to TCT, and MAX-EH converges to POWMU for higher values of $P_{A}$. Similar behaviour is observed for networks with larger number of users.

Fig. 3 indicates the schedule length of a 5 -user network for varying $P^{\max }$ values. POWMU outperforms TCT up to $6.8 \%$,

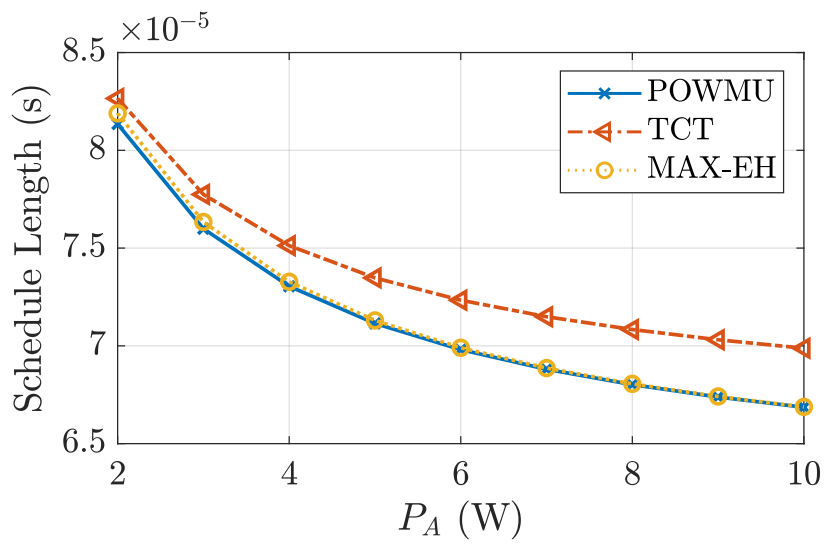

Fig. 2. Schedule length (s) vs. transmission power (W) of AP for a 5-user network.

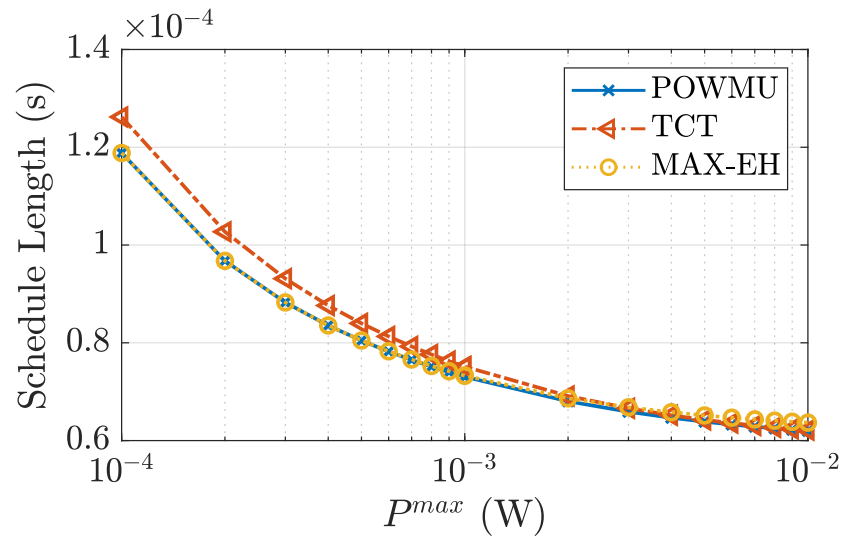

Fig. 3. Schedule length (s) vs. the maximum UL transmit power (W) for a 5-user network

especially for smaller $P^{\max }$. As we loosen that constraint, TCT converges to our solution. It is as expected, since we optimize the time allocations considering the transmit power limitation, while, if allowed, TCT tends to assign higher transmit power to the users for faster IT, compromising $\mathrm{EH}$ length in favor of a lower total schedule length. Forcing TCT to use $P^{\max }$ may result with increased EH length but not improved IT length. MAX-EH performs closely to the optimum, especially for lower $P^{\max }$.

Fig. 4 presents the schedule length for varying number of users, from 10 to 25. POWMU reduces TCT's solution up to $2.9 \%$; whereas MAX-EH performs closely to POWMU.

In Fig. 5, run-times of the algorithms are compared for different number of user, ranging from 1 to 25. Since MAX$\mathrm{EH}$ is a linear time algorithm, it performs significantly better than logarithmic time POWMU and TCT, especially for largesize networks. Likewise, POWMU surpasses TCT as predicted, considering our algorithm performs the optimal $\tau_{0}$ search on a narrower interval. POWMU and MAX-EH reduce TCT's runtime $71.5 \%$ and $84.1 \%$ for $K=25$, respectively. 


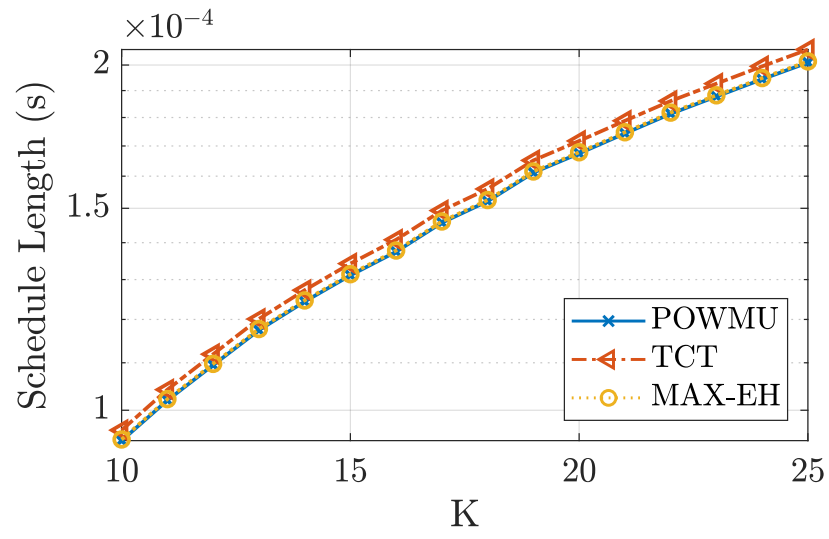

Fig. 4. Schedule length (s) vs. the number of the users.

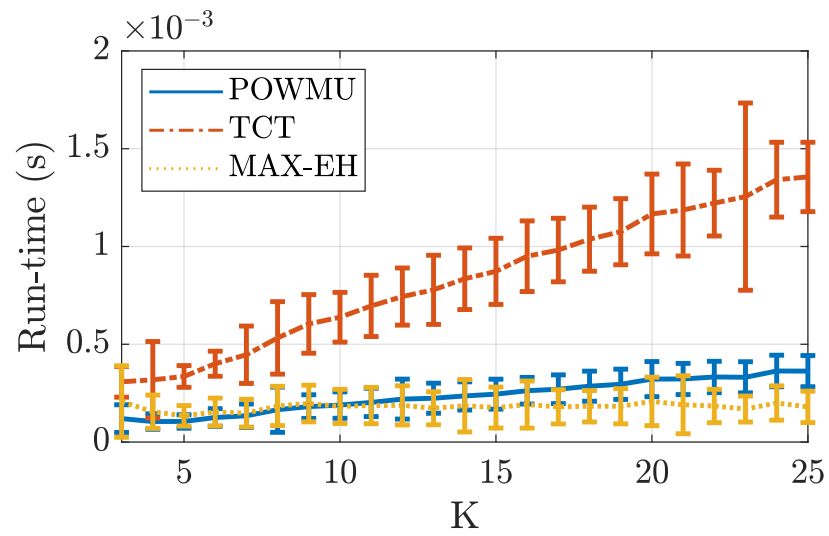

Fig. 5. Run time vs the number of users.

\section{CONCLUSION}

We analyzed the minimum length scheduling problem for a HTT network with a half-duplex single antenna AP, and multiple, single antenna users. The EH and IT times, and UL transmit powers are allocated to optimize the scheduling length under minimum data and maximum transmit power limitations of the users. The problem is proven to be nonlinear and non-convex. We first transformed this problem into a bi-level optimization problem, where we exploited the convexity of the subproblems. We derived an expression for the IT lengths as a function of EH length, and then, searched for the optimal $\mathrm{EH}$ length that minimizes the total length. An algorithm, POWMU, that provides a near optimal solution within an error bound is proposed. Further, we presented a suboptimal algorithm, MAX$\mathrm{EH}$, which exploits the features of the single user network to solve the multiple user network. Our algorithms are compared with a benchmark algorithm, TCT, which does not regard the UL transmit power limit. Simulation results showed that POWMU and MAX-EH outperform TCT under tight power conditions and their run-times are significantly better, especially for larger networks.

\section{REFERENCES}

[1] A. Osseiran, V. Braun, T. Hidekazu, P. Marsch, H. Schotten, H. Tullberg, M. A. Uusitalo, and M. Schellman, "The foundation of the mobile and wireless communications system for 2020 and beyond: Challenges, enablers and technology solutions," in 2013 IEEE 77th Vehicular Technology Conference (VTC Spring), June 2013, pp. 1-5.

[2] H. Ju and R. Zhang, "Throughput maximization in wireless powered communication networks," IEEE Transactions on Wireless Communications, vol. 13, no. 1, pp. 418-428, January 2014.

[3] _ _ "Optimal resource allocation in full-duplex wireless-powered communication network," IEEE Transactions on Communications, vol. 62, no. 10, pp. 3528-3540, Oct 2014.

[4] L. Liu, R. Zhang, and K. Chua, "Multi-antenna wireless powered communication with energy beamforming," IEEE Transactions on Communications, vol. 62, no. 12, pp. 4349-4361, Dec 2014.

[5] Z. Chu, F. Zhou, Z. Zhu, R. Q. Hu, and P. Xiao, "Wireless powered sensor networks for internet of things: Maximum throughput and optimal power allocation," IEEE Internet of Things Journal, vol. 5, no. 1, pp. 310-321, Feb 2018

[6] X. Chen, X. Wang, and X. Chen, "Energy-efficient optimization for wireless information and power transfer in large-scale mimo systems employing energy beamforming," IEEE Wireless Communications Letters, vol. 2, no. 6, pp. 667-670, December 2013

[7] F. Shan, J. Luo, W. Wu, and X. Shen, "Delay minimization for data transmission in wireless power transfer systems," IEEE Journal on Selected Areas in Communications, vol. 37, no. 2, pp. 298-312, Feb 2019.

[8] X. Kang, C. K. Ho, and S. Sun, "Full-duplex wireless-powered communication network with energy causality," IEEE Transactions on Wireless Communications, vol. 14, no. 10, pp. 5539-5551, Oct 2015.

[9] J. Wang, X. Kang, Y. Liang, and S. Sun, "An energy harvesting chain model for wireless-powered IoT networks," in 2018 10th International Conference on Wireless Communications and Signal Processing (WCSP), Oct 2018, pp. 1-6.

[10] K. Chi, Y. Zhu, Y. Li, L. Huang, and M. Xia, "Minimization of transmission completion time in wireless powered communication networks," IEEE Internet of Things Journal, vol. 4, no. 5, pp. 1671-1683, Oct 2017.

[11] D. P. Bertsekas, "Convexification procedures and decomposition methods for nonconvex optimization problems," Journal of Optimization Theory and Applications, vol. 29, no. 2, pp. 169-197, Oct 1979. [Online]. Available: https://doi.org/10.1007/BF00937167

[12] F. S. Hillier and G. J. Lieberman, Introduction to Operations Research, 9th Ed. New York: McGrawHill, 2010.

[13] C. Zalinescu, Convex Analysis in General Vector Spaces. River Edge, N.J.: World Scientific, 2002.

[14] E. W. Weisstein. Bisection. MathWorld - A Wolfram Web Resource. Accessed: 2019-03-12. [Online]. Available: http://mathworld.wolfram.com/Bisection.html

[15] S. Y. Seidel and T. S. Rappaport, "914 MHz path loss prediction models for indoor wireless communications in multifloored buildings," IEEE Transactions on Antennas and Propagation, vol. 40, no. 2, pp. 207-217, Feb 1992.

[16] R. Morsi, D. S. Michalopoulos, and R. Schober, "Multiuser scheduling schemes for simultaneous wireless information and power transfer over fading channels," IEEE Transactions on Wireless Communications, vol. 14, no. 4, pp. 1967-1982, April 2015. 